\title{
Tendinopatias, Smartphones e dispositivos eletrônicos: relato de quatro casos
}

Tendinopathies, Smartphones and electronic devices: report of four cases

Tendinopatías, Smartphones y dispositivos electrónicos: reporte de cuatro casos

Luis Guilherme Rosifini Alves REZENDE

Médico Assistente da Cirurgião de Mão do Hospital das Clínicas da Faculdade de Medicina de Ribeirão Preto da Universidade de São Paulo (HC-FMRP-USP)

https://orcid.org/0000-0002-2037-0135

Filipe Jun SHIMAOKA

Médico Assistente da Cirurgião de Mão do Hospital das Clínicas da Faculdade de Medicina de Ribeirão Preto da Universidade de São Paulo (HC-FMRP-USP) https://orcid.org/0000-0002-1475-1396

Gunter Shin Iti Takamori SHIMABUKURO

Médico Assistente da Cirurgião de Mão do Hospital Estadual de Serrana do Complexo do Hospital das Clínicas da Faculdade de Medicina de Ribeirão Preto da Universidade de São Paulo

Luiz Garcia MANDARANO-FILHO

Médico Assistente da Cirurgião de Mão do Hospital das Clínicas da Faculdade de Medicina de Ribeirão Preto da Universidade de São Paulo (HC-FMRP-USP) https://orcid.org/0000-0002-2774-9471

Nilton MAZZER

Professor Titular e Chefe da Divisão de Cirurgia da Mão do Hospital das Clínicas da Faculdade de Medicina de Ribeirão Preto da Universidade de São Paulo (HC-FMRP-USP) https://orcid.org/0000-0002-1239-7602

Cláudio Henrique BARBIERI

Professor Titular, Faculdade de Medicina de Ribeirão Preto da Universidade de São Paulo (FMRP - USP) https://orcid.org/0000-0003-3858-602X

\section{Resumo}

O aumento do uso de celulares do tipo smartphones e de dispositivos eletrônicos secundários ao aumento das atividades do tipo home office e atividades remotas gerou um aumento nas queixas de dores nas mãos durante o último ano. Isto se encontra relacionado ao aumento do tempo de uso destes dispositivos. Apresentamos uma série de quatro casos de pacientes com queixas de dores nas mãos associados ao uso de dispositivos eletrônicos e a forma como foram manejados, bem como uma revisão sobre a literatura disponível sobre estas afecções, com o objetivo de conscientizar os autores sobre uma possível correlação das queixas de dores nas mãos associadas ao uso excessivo de dispositivos eletrônicos.

Descritores: Tendinopatia; Smartphone; Traumatismos da Mão.

\section{Abstract}

The increase in the use of smartphone-type cell phones and electronic devices secondary to the increase in the home office and remote activities has led to an increase in complaints of hand pain over the last year. It's associated with the overused time of these devices. We present a series of four cases of patients complaining of hand pain associated with electronic devices and how they handled them. A review of the available literature on these conditions makes the authors aware of a possible correlation and the complaints of pain in the hands associated with excessive use of electronic devices.

Descriptors: Tendinopathy; Smartphone; Hand Injuries.

\section{Resumen}

El aumento en el uso de teléfonos móviles tipo smartphone y dispositivos electrónicos como consecuencia del aumento de la oficina en casa y las actividades remotas ha provocado un aumento de las quejas de dolor de manos durante el último año. Esto está relacionado con el mayor tiempo de uso de estos dispositivos. Presentamos una serie de cuatro casos de pacientes que se quejan de dolor en la mano asociado al uso de dispositivos electrónicos y su manejo, así como una revisión de la literatura disponible sobre estas condiciones, con el objetivo de concienciar a los autores de un posible correlación quejas de dolor en las manos asociadas con el uso excesivo de dispositivos electrónicos.

Descriptores: Tendinopatía; Teléfono Inteligente; Traumatismos de la Mano.

\section{INTRODUÇÃO}

As dores no punho secundárias ao uso de dispositivos eletrônicos apresentaram um aumento expressivo no último ano, durante a pandemia, devido aumento das atividades do tipo homeoffice. Isto se dá ao fato do aumento do tempo de uso de celulares do tipo smartphones, tablets, notebooks, computadores, dentre outros dispositivos tecnológicos. Desta forma, os pacientes passaram a utilizar por um período maior do que - habitual para realizar suas funções de trabalho. Além disso, estes dispositivos eletrônicos também são utilizados como meios de comunicação, ferramentas voltadas para 0 ensino e métodos de lazer e outras formas acessórias, como a função GPS e de máquina fotográfica, por exemplo ${ }^{\hat{1}, 2}$.
Alguns estudos têm reportado queixas como DeQuervain, tendinopatias e até degeneração das articulações do polegar, dedos e punho pelo uso excessivo destes dispositivos. Porém, sintomas variados foram descritos para estas associações ${ }^{3-5}$.

Nestas situações, estas afecções podem enquadrar-se como doenças ocupacionais, conhecidas em nosso meio como LER (Lesões por Esforço Repetitivo) ou DORT (Distúrbios Osteomusculares Relacionados ao Trabalho), que são um conjunto de afeções que atingem principalmente os membros superiores.

No presente estudo, relatamos quatro casos de pacientes com diferentes tipos de tendinopatias pelo uso de dispositivos eletrônicos. 


\section{CASO CLÍNICO}

\section{- Caso 1}

Paciente do sexo masculino, 21 anos de idade, destro, secretário adjunto da prefeitura municipal, apresentando queixas de dores nas mãos, piores a esquerda, há 7 meses, após o início de suas atividades do tipo homeoffice. Refere que passou a utilizar durante mais tempo seus dispositivos eletrônicos, aumentando a frequência de trabalho, atingindo até 12 horas de uso contínuo diário. Não possui comorbidades ou transtornos aditivos e não realiza atividades físicas. Ao exame apresentava dor difusa na região dorsal dos dedos ao nível das interfalangeanas proximais e metacarpofalangeanas e discreto edema na mão esquerda, conforme Figura $1 \mathrm{~A}$. Apresentava dores para a palpação do polegar e teste de Finkelstein e Eichhoff positivos. O ultrassom confirmou 0 diagnóstico de Tenossinovite estenosante de De Quervain, porém, sem alterações na região extensora dos dedos. Não havia alterações nas radiografias. Optou-se por tratamento conservador com analgesia, anti-inflamatórios, órtese para polegar, fisioterapia e implementação de intervalos durante os períodos de uso dos dispositivos eletrônicos. Após quatro meses, o paciente retornou com redução dos sintomas, mas com persistência das dores no polegar no exame físico, sendo optado por infiltração do primeiro compartimento extensor com uma solução de corticoide associado a lidocaína. Após, o paciente evoluiu com resolução completa dos sintomas.

\section{Caso 2}

Paciente do sexo masculino, 42 anos, destro, professor universitário há 15 anos. Refere queixas de dores na região dos polegares há 9 meses, desde o início das atividades escolares do tipo remotas para ensino. Refere que houve um aumento expressivo em sua demanda de trabalho, pois houve a necessidade de gravações de aulas e implemento de novas tecnologias para ensino à distância de seus alunos. É hipertenso, controlado com uso de Losartana, e não apresenta outras comorbidades ou transtornos aditivos, como etilismo, tabagismo ou uso de medicações. Não realiza atividades físicas ou possuía queixas similares no passado. Quando questionado, referia utilizar os dispositivos eletrônicos por pelo menos 10 horas por dia. Ao exame, apresenta dor a palpação do primeiro compartimento extensor dos polegares, com testes de Finkelstein e Eichhoff positivos, piores à direita (Figura 1B). $O$ ultrassom confirmou 0 diagnóstico de Tenossinovite Estenosante de De Quervain bilateral, sem anomalias nos tendões, septações no túnel ou cistos e tumores. Optou-se pelo tratamento conservador com analgesia, AINES, uso de órtese para o polegar e fisioterapia para alongamentos e fortalecimento das musculaturas. $O$ paciente foi orientado a reduzir o tempo do uso de celular, bem como adicionar intervalos de 10 minutos a cada 2 horas de uso dos dispositivos eletrônicos, apresentando resolução completa dos sintomas.

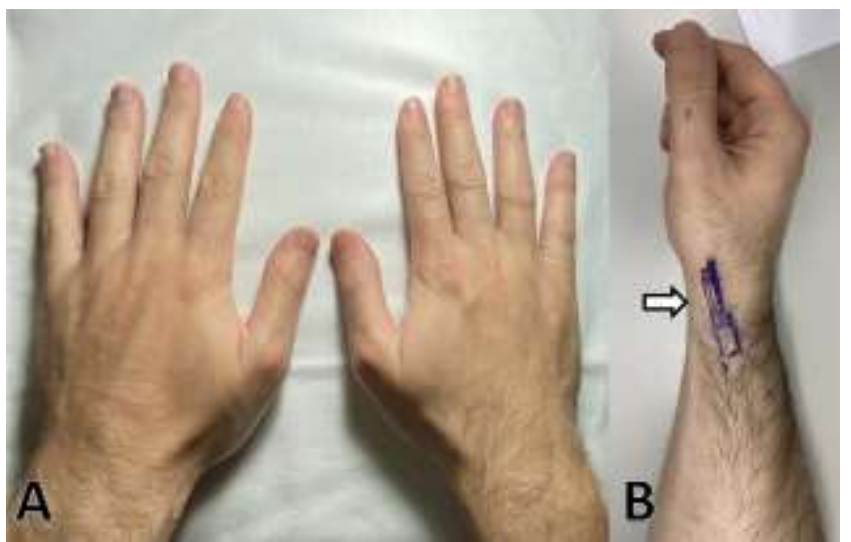

Figura 1. A: Caso no1, edema dorsal na região dos dedos da mão esquerda. B: Caso №2, região dolorosa (seta) e anatomia da região do primeiro compartimento extensor (roxo).

- Caso 3

Paciente do sexo feminino, 19 anos, destra, digital influencer, apresentando queixas de dores na região do polegar esquerdo e dor na região ulnar do punho esquerdo desde o lockdown em sua cidade, há 1 ano. Refere que aumentou seu número de stories e postagens nas mídias das redes sociais, compartilhando dicas de sua vida durante a pandemia, tendo em vista a redução das festas e eventos sociais presenciais. Realiza Yoga e atividades físicas diariamente com seu Personal Trainer, e não apresentava queixas similares no passado. A paciente é vegetariana, não possui comorbidades, transtornos aditivos e não realiza uso de medicamentos. Ao exame, observou-se dor a palpação da base do polegar e do primeiro compartimento extensor (Figura 2A). Os testes de Finkelstein e Eichhoff foram positivos. A ultrassonografia confirmou 0 diagnóstico de tenossinovite estenosante de De Quervain associada a inflamação na primeira articulação carpometacarpal do polegar esquerdo. Não havia alterações radiográficas. Optou-se por tratamento conservador com analgesia, fisioterapia e órtese para polegar, bem como aumento do tempo de intervalo no uso de smartphones. Após a reavaliação em 3 meses, houve melhora das queixas com resolução completa dos sintomas. 


\section{- Caso n4}

Paciente do sexo feminino, 32 anos, destra, educadora físico e professora de pósgraduação. Refere dor difusa no punho e polegar esquerdo, após início de atividades remotas de ensino há 6 meses. Refere que aumentou o uso de seu smartphone e tablet para suas aulas de pós-graduação, atingindo uma média até 10 horas por dia de uso. Ao exame, observa-se dor na região ulnar punho, principalmente na região da fóvea com sinal da fóvea positivo. $O$ exame físico também foi positivo para De Quervain (Figura 2B). Não apresentou alterações nos exames radiográficos, porém a Ressonância Magnética apresentou alteração de Sinal na Fóvea. Optouse por tratamento conservador com fisioterapia e intervalos no uso dos dispositivos eletrônicos, obtendo-se boa resposta, com resolução dos sintomas de De Quervain após dois meses, e resolução das queixas da dor ulnar do punho após quatro meses.

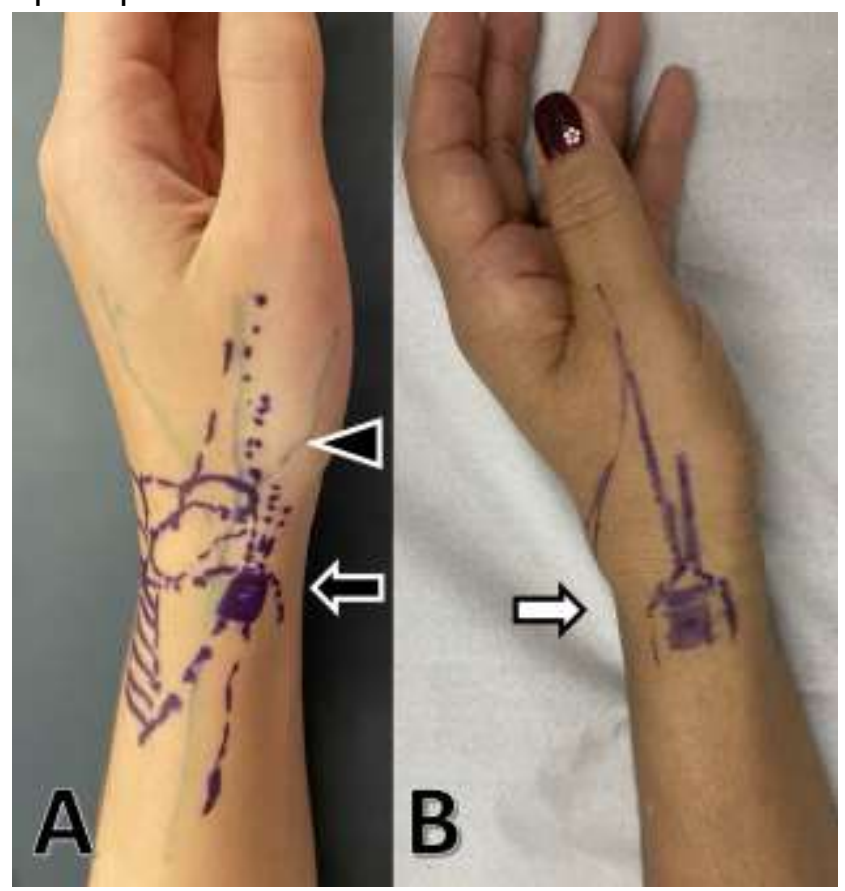

Figura 2. A: Caso ํㅜ: cabeça de seta dor na base do polegar, seta indica dor na região do $1^{\circ}$ compartimento extensor e desenho anatômico utilizado para explicar diagnósticos de dor no paciente. B: Caso n4: seta indica local de dor no $1^{\circ}$ compartimento extensor.

DISCUSSÃO

O aumento das atividades do tipo homeoffice e atividades remotas após o início da pandemia, fez com que houvesse um aumento do tempo de uso de dispositivos eletrônicos. Desta forma, os pacientes passaram a apresentar queixas de dores nas mãos, principalmente na região da base do polegar. Dores nas regiões ulnares do punho também foram relatadas. Em nossa série de casos, apresentamos pacientes com queixas de dores na região do polegar, que foram manejados de forma conservadora, obtendo-se um bom resultado, exceto por um dos pacientes, que necessitou de infiltração no primeiro compartimento extensor ${ }^{1-7}$.

Com o aumento do uso dos dispositivos eletrônicos, houve um aumento do uso da musculatura e das pequenas articulações das mãos, que passaram a receber uma sobrecarga cíclica excessiva, com um período de repouso menor do que o habitual. As queixas variam de tendinopatias do polegar, como tenossinovite de De Quervain, até queixas de artrite da primeira articulação carpometacarpal e queixas de dores ulnares do carpo ${ }^{1-9}$.

Existem duas posições amplamente utilizadas para o uso de smartphones. A mais comum é o uso dos smartphones em ambas as mãos, realizando uma extensão associada ao desvio ulnar do punho, com uso dos polegares para digitação e toque da tela. Esta posição é comumente referida pelos pacientes para digitação rápida ou para uso de jogos nos smartphones. A segunda posição consiste no apoio do celular por uma das mãos, realizando o "toque da tela" pelo polegar ou dedos. É utilizada para digitação "monogidital", desbloqueio de tela, toques específicos, entre outras funções (Figura 3).

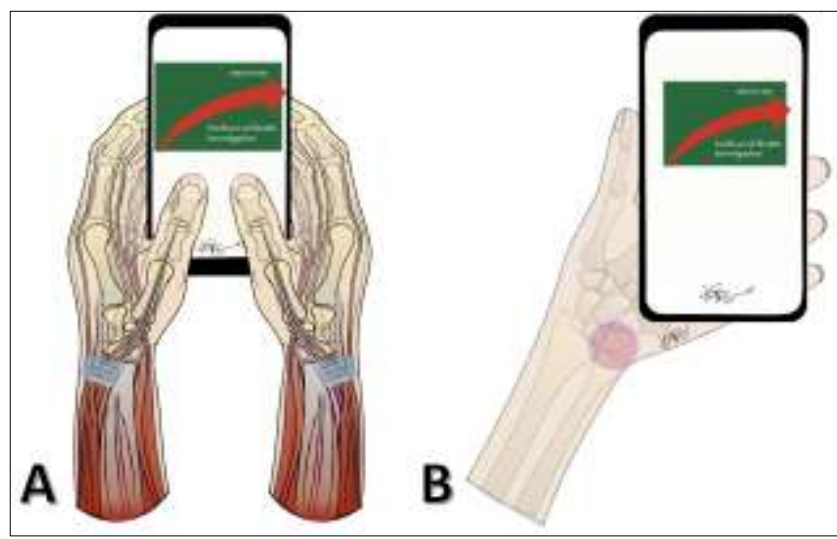

Figura 3. Posições para digitação do celular. A: Tendões destacados em roxo e rosa na mão direita representam os tendões do primeiro compartimento extensor, acometidos pelo excesso de digitação. B: Região em roxo determina impacto na região ulnar do punho, comuns nas posições para segurar o celular, enquanto a outra mão realiza a digitação monodigital (Fonte: Luis Guilherme Rosifini Alves Rezende, 2021).

A estratégia de adicionar intervalos que fiquem longe dos dispositivos eletrônicos ao longo do dia, pretende gerar descanso para estas musculaturas e pequenas articulações das mãos, com a finalidade de tentar reduzir o estresse cíclico secundário ao seu uso diário. É importante orientar o paciente que, durante os intervalos, deve-se ficar afastado dos dispositivos eletrônicos, tendo em vista que, as queixas encontram-se associadas aos movimentos repetitivos de digitação e postura 
viciosa da mão, punho e dedos. No período do intervalo, aconselhamos os pacientes a buscar outras atividades, que permitam repouso das mãos, punho e dedos e evitar o uso das mesmas durante.

Além disso, um bom fortalecimento realizado pelo terapeuta da mão ou fisioterapeuta, permite que haja uma hipertrofia desta musculatura e um melhor preparo articular, de forma que a mão poderá suportar esta carga cíclica do trabalho, reduzindo a frequência, duração e intensidade dos sintomas. O fortalecimento gera um estímulo biológico programado para a musculatura e articulação, desencadeando respostas metabólicas osteomusculares benéficas..$^{5-9} \mathrm{Na}$ ausência de sua melhora com o tratamento conservador, a infiltração é um método que pode ser utilizado para o tratamento da tenossinovite de De Quervain. ${ }^{10}$ Além disso, diversos métodos ergonômicos podem ser utilizados, além da correção da má-postura, frequentemente associada a queixas no trabalho.

CONSIDERAÇÕES FINAIS

Acreditamos que o aumento no tempo de uso dos dispositivos eletrônicos durante a pandemia, secundário ao aumento da carga horária de trabalho do tipo homeoffice e atividades remotas encontra-se relacionado ao aumento das queixas de dores nas mãos e punhos. Desta forma, estratégias de adicionar intervalos ao longo do dia, permitindo o repouso das mãos, punho e dedos, porém reduzir as queixas e sintomas relacionados a estas afecções.

REFERÊNCIAS

1. Toh SH, Coenen P, Howie EK, Straker LM. The associations of mobile touch screen device use with musculoskeletal symptoms and exposures: A systematic review. PLoS One. 2017;12(8): 1-22.

2. Amjad $F$, Farooq $M N$, Batool $R$, Irshad $A$. Frequency of wrist pain and its associated risk factors in students using mobile phones. Pak J Med Sci. 2020;36(4):746-49.

3. Canillas F, Colino A, Menéndez P. Cellular Phone Overuse as A Cause for Trapeziometacarpal Osteoarthritis: A Two Case Report. J Orthop Case Rep. 2014;4(4):6-8.

4. Iwata K. Smartphone-induced tendinitis: A case report. J Family Med Prim Care. 2019 May;8(5):1784-85.

5. Eitivipart AC, Viriyarojanakul S, Redhead L. Musculoskeletal disorder and pain associated with smartphone use: A systematic review of biomechanical evidence. Hong Kong Physiother J. 2018;38(2):77-90.
6. Fernandez-Guerrero, IM. "WhatsAppitis".The Lancet, 2014; 383(9922):1040

7. Ali M, Asim M, Danish SH, Ahmad F, lqbal A, Hasan SD. Frequency of De Quervain's tenosynovitis and its association with SMS texting. Muscles Ligaments Tendons J. 2014;4(1):74-8.

8. Alsalameh AM, Harisi MJ, Alduayji MA, Almutham AA, Mahmood FM. Evaluating the relationship between smartphone addiction/overuse and musculoskeletal pain among medical students at Qassim University. J Family Med Prim Care. 2019;8(9):2953-59.

9. Sharan D, Ajeesh PS. Risk factors and clinical features of text message injuries. Work. 2012;41(1):1145-48.

10. Vasconcelos BM, Rezende LGRA, Chamon $H G$, Vilela LH. Efetividade da infiltração de corticosteroides no tratamento de pacientes com tenossinovite estenosante de De Quervain: resultado terapêutico. Arch Health Invest. 2019;8(5):245-50.

\section{CONFLITO DE INTERESSES}

Os autores declaram não haver conflitos de interesse

\section{AUTOR PARA CORRESPONDÊNCIA}

\section{Luis Guilherme Rosifini Alves Rezende \\ Av. Bandeirantes, 3900 - Vila Monte Alegre, 14049-900 Ribeirão Preto - SP, Brasil \\ E-mail: Igrezende@usp.br}

